\title{
Tangence
}

\section{Le pluriel intérieur}

\section{Jaqueline Rousseau-Dujardin}

Numéro 42, décembre 1993

Le récit de soi

URI : https://id.erudit.org/iderudit/025789ar

DOI : https://doi.org/10.7202/025789ar

Aller au sommaire du numéro

Éditeur(s)

Tangence

ISSN

0226-9554 (imprimé)

1710-0305 (numérique)

Découvrir la revue

Citer cet article

Rousseau-Dujardin, J. (1993). Le pluriel intérieur. Tangence, (42), 68-79.

https://doi.org/10.7202/025789ar d'utilisation que vous pouvez consulter en ligne.

https://apropos.erudit.org/fr/usagers/politique-dutilisation/ 


\section{Le pluriel intérieur \\ Jacqueline Rousseau-Dujardin}

C'est un curieux statut que celui du psychanalyste: compte tenu de la nécessité d'une analyse personnelle, clé de voûte de sa "formation", il s'est "récité " (et aussi re-cité, oh combien!) pendant les années passées sur le divan. Oralement, bien entendu, puisqu'il s'agit d'une talking cure. Le voilà désormais dans le fauteuil en admettant qu'il en ait fini, provisoirement en tout cas, avec le divan. Du coup, le "récit de soi" devient hasardeux: difficile avec ses proches, surtout s'ils n'ont pas partagé la même expérience et n'ont pas perçu l'infinie complexité du récit en question; déontologiquement et techniquement déconseillé en public, pour cause de nécessaire disponibilité au transfert. L'analyste doit rester autant que possible, on le sait, une sorte de "figure blanche" sur laquelle viendront se jouer et s'imprimer les images projetées par ses patients. Passe encore qu'il paraisse en des lieux scientifiques pour exposer ses conceptions psychanalytiques avec toute la neutralité présumée du chercheur. Ses patients, pour peu qu'ils soient eux-mêmes "psy", hésiteront cependant à aller l'entendre: leur propre "récit de soi" se trouvera-t-il aussi libre après la circonstance au cours de laquelle des bribes du "soi" de leur analyste se laisseront saisir, quoi qu'il en ait? Pourtant beaucoup sautent le pas, surtout après quelques années d'analyse: le transfert est assez bien accroché (comme on le dit d'un foetus) et résistera à l'épreuve. L'occasion fournira même peut-être du "matériel". La part d'énigme et d'interprétation restera large, la place libre pour le fantasme. D'autant que le psychanalyste, dans ces circonstances, ne se raconte pas: il donne sa personne à voir avec ce qu'elle peut révéler de l'histoire de sa vie compte tenu de tout le hasardeux de l'entreprise de déchiffrement ainsi consentie à l'autre, l'auditoire. Et il dit ce qu'il pense, dans le domaine de la connaissance, s'entend. Non sans prendre le risque de se voir dévoilé en tant que personne à travers les notions exposées; rapport de l'homme à l'œuvre in situ, in statu nascendi. "Voilà ce que je pense, vous pouvez en déduire ce que je suis. "Mais en ce sens, "récit de soi " prend une acception trop large: toute présence à l'autre en constituerait un. 
Ce n'est pas si faux d'ailleurs puisque c'est sur ces apparitions, ces présences, que l'autre va établir son récit de vous, et que ces récits d'origine différente ont inévitablement des points de correspondance. Mais il faut bien des limites au genre: tout n'est pas dans tout. Admettons que le "récit de soi " nécessite de la part de "soi " une décision de s'y engager.

L'écriture du récit de soi est une autre affaire. Si l'on en éprouve la tentation tout en étant psychanalyste, ce ne sont pas les précédents qui manquent pour vous justifier, qu'ils soient lointains et même psychanalytiquement originels (Freud bien entendu) ou plus proches (D. Anzieu, J.B. Pontalis pour n'évoquer que les plus voisins dans l'espace). Mais le régime de ces écrits varie à l'infini: entreprise "simplement "auto-biographique comme L'amour des commencements de J.B. Pontalis ${ }^{1}$, par exemple, mais, bien avant, comme l'auto-biographie d'Hélène Deutsch $^{2}$. Ou bien décor rapidement brossé où situer l'œuvre et éclairer l'histoire de la discipline pour "l'auto-présentation de Freud" 3 . Tressage historico-auto-analytique dans tel ou tel ouvrage de Theodor Reik, qu'il s'agisse de Variations psychanalytiques sur un thème de Mabler ou de Fragments d'une grande confession ${ }^{4}$. Bien entendu, déguisement en matériel émanant d'un patient de ce qui vient de l'analyste lui-même comme on le voit tout au long de l'ouvrage psychanalytique princeps qu'est L'interprétation des rêves. Journaux intimes pré- ou postanalytiques comme celui de Marie Bonaparte 5 . Plus curieux, écrits quotidiens du patient relatant l'analyse dans le temps même où elle se fait (comme le livre de Hilda Doolittle, Visage de Freud $^{6}$ ). Et puis, bien sûr les récits d'analyse a posteriori dont les quelques cas publiés ne constituent probablement que la partie

1 J.B. Pontalis, L'amour des commencements, Paris, Gallimard, 1986.

2 Helen Deutsch, Confrontation with myself, New York, Norton, 1973. Trad. franç.: Autobiographie, Paris, Mercure de France, 1980.

3 Voir à ce sujet Jean-François Chiantaretto, "Freud auto-biographe ou l'autobiographie par excès ", Paris, Le Coq-Héron, 1992.

4 Theodor Reik, Fragment of a great confession. A psychoanalytical biograpby, New York, Farrar er Strauss, 1949. Trad. franç.: Fragment d'une grande confession, Denoël, Paris, 1973; Variations psychanalytiques sur un thème de Gustav Mabler. Extrait en trad. franç. de The Haunting Melody, Paris, Denoël, 1973.

5 Marie Bonaparte, À la mémoire des disparus, Paris, PUF, 1953.

6 Hilda Doolittle, Tribute to Freud, Pearson, 1956. Trad. franç.: Visage de Freud, Paris, Denoël, 1977. 
émergée d'un considérable iceberg, et dans lesquels le "récit de soi" s'abrite derrière l'exposé d'une expérience quasi scientifique. Au demeurant, tous les narrateurs d'analyse ne sont pas devenus eux-mêmes psychanalystes... Reste d'ailleurs, pour ceux qui ont choisi cette voie, la possibilité de prendre ce qu'on pourrait nommer la "tangente biographique", en se racontant à travers ce qu'ils ont vu de la vie de l'autre, tel Jones appliqué au récit de la saga freudienne, et impliqué, confondu presque, dans son œuvre à tel point que, comme le dit Bruno Bettelheim: "Tandis qu'il travaillait au dernier volume, Jones savait que sa fin approchait. Il luttait donc contre le temps, et sa mort suivit de peu le moment où il put écrire le mot "fin"., 7

Quoiqu'il en soit, les psychanalystes ont, comme leurs patients, à gérer un certain silence, celui de l'interruption du récit de soi poursuivi oralement pendant des années sur le divan. Avec cette différence cependant que s'ouvrent devant eux les espaces théoriques où ils pourront, s'ils le désirent, parler ou écrire moyennant un changement de leur dispositif transférentiel - le passage à ce qu'on a pu nommer un transfert sur la théorie théorie comprise au sens large du terme, étant donné le statut épistémologique de la psychanalyse. C'est probablement façon de donner le change à la solitude post-analytique, d'accomplir le nécessaire processus de deuil qui accompagne la sortie, ne seraitce que provisoire, du divan, d'aider à ce qu'on appelle vilainement la "liquidation du transfert". Nulle raison, à ma connaissance, ne rend entièrement compte du fait que, si pour certains, l'écriture vient remplacer ou poursuivre l'analyse, pour d'autres, elle n'apparaît pas utile ou nécessaire. Mais on ne saurait négliger l'idée que l'écriture "scientifique", si elle s'avance masquée, est encore récit de soi, d'une part de soi, même si elle en constitue un genre particulier.

C'est une idée moderne, je veux dire de la modernité. Et qui n'est sans doute pas admise par tous, même si déjà nous participons de ce qui a été désigné comme post-modernité. Certes, il est pour le moins désagréable que la "sincérité" soit systématiquement soupçonnée. Mais accepter l'ex-centrement du sujet théorisant est encore plus difficile, voire scandaleux, que celui du

7 Bruno Bettelheim, "Deux regards sur Freud", dans Le poids d'une vie, Paris, Laffont, 1989 pour la trad. franç., p. 61. 
sujet tout court, si j'ose dire et c'est alors que se ravive le plus douloureusement la troisième blessure narcissique décrite par Freud: l'homme n'est qu'illusoirement maître chez lui, sa conscience est traversée par des courants inconscients, sa pensée rationnelle travaillée par des filons d'irrationnel dont il a peur d'apercevoir la richesse. N'est-il pas frappant, par exemple, de discerner, à la naissance d'une œuvre, d'un ensemble d'idées qui dans certains cas deviendra un système de pensée ou un style artistique, de discerner donc dans quel terreau s'enracine le processus créateur, de qui il consent à se reconnaître l'héritier, par rapport à qui il accepte de se situer débiteur. Certains même dressent la liste de leur ascendance intellectuelle ou artistique, comme le fit entre autres le compositeur Arnold Schoenberg ${ }^{8}$. Ils pourraient tout aussi bien dire, et ils le font parfois, encore que ce soit sans doute plus difficile, contre qui leur "nouveauté" s'est constituée, ils pourraient nommer ceux qu'ils veulent atteindre. Cette généalogie élue, à l'opposé de celle que le sort vous a imposée, ne voit-on pas de quels fantasmes elle est pénétrée, comment la lignée des ancêtres choisis est tressée, quelle que soit leur valeur objective, avec l'élaboration du roman familial? Comment elle vise à panser une autre blessure narcissique: celle de n'avoir été pour rien dans sa propre naissance? Et n'apparaîtelle pas à ce titre comme un chapitre, et non des moindres, du récit de soi?

On devine que, pour ce qui en est des psychanalystes, le transfert est de nature à accentuer cet aspect des choses. La psychanalyse apparaît en effet comme le processus d'une nouvelle gestation tout en remettant en lumière ce qui a abouti à la conception, à la naissance "réelle" et aux premières années de formation de l'individu. Elle propose à celui-ci, en somme, d'accepter son origine dite naturelle, de ne pas consacrer l'essentiel de son énergie à s'en construire une autre dans l'imaginaire; et, en même temps, de participer activement à une reformulation de sa personne, avec les conséquences sur ses actes de la vue nouvelle qu'il acquiert de lui. De quoi certes, modifier le récit de soi de tout analysant: ainsi voit-on en cours de cure les imagos qui peuplaient son monde intérieur prendre d'autres silhouettes, d'autres consistances, ses dispositions propres changer à leur

8 Voir la préface de Chemins vers la Nouvelle Musique, d'Anton Webern, Paris, Lattès, 1980. 
72

égard. Q'un reflet de son analyste s'y discerne, en rapport avec le transfert, et même si l'identification est loin d'occuper celui-ci tout entier, c'est probable. Mais qui le sait s'il s'agit d'une analyse dite thérapeutique? Dans les milieux psychanalytiques, au contraire, qui sont autant de bouillons de culture (à bien des sens du terme), les transformations se repèrent, les influences se marquent, parfois dans les personnes (le mimétisme lacanien était tel en son temps qu'on ne voit pas très bien comment Lacan aurait été évincé de tel ou tel récit de soi sans un aveuglement qui eût mal convenu, justement, aux analystes). Mais aussi, elles se retrouvent dans les œuvres quand œuvre il y a: ainsi discernerat-on Ferenczi dans le textes de Mélanie Klein, Mélanie Klein dans ceux de Winnicott qu'elle n'a, il est vrai que "contrôlé", ou, plus près de nous, Jacques Lacan chez Serge Leclaire. Traces que l'on n'attribuera pas seulement à certaines rencontres de pensée mais aux restes transférentiels instituant une nouvelle parenté, souvent compliquée si l'on tient compte des "tranches d'analyse", parenté à laquelle le seul Freud échappe puisque, inventeur de la psychanalyse, il n'a pas eu d'analyste ou d'analystes. Quoi qu'on en ait, il s'agit d'un récit de soi plus ou moins latent, mais, on l'espère pour la psychanalyse, plus reconnu qu'ailleurs à travers le grillage de la création théorique.

Il faudrair d'ailleurs condidérer de plus près à cet égard les écrits psychanalytiques dans leur forme la plus répandue, c'est-àdire la narration de "cas" assortie de développements d'allure scientifique ("d'allure" étant donné le statut épistémologique incertain de la psychanalyse). J'évoquais plus haut la "tangente" biographique, hétéro-biographique pourrait-on dire adoptée par certains, Jones entre autres, pour déguiser peut-être le récit de soi. Mais ces narrations de la personne, de la vie de tel ou tel patient particulièrement intéressant, ne répondent-elles pas, dans une certaine mesure, au même désir de se raconter "par la bande"? De tourner la frustration apportée par la fin de l'analyse qui coupe court, même après de longues années, à ce luxe d'une parole sur soi souvent appuyée sur des écrits intimes? Ces considérations n'aideraient-elles pas à comprendre pourquoi les auteurs de ces récits de l'autre, Freud tout le premier, et malgré ses précautions, prennent leur parti sans trop de difficultés, semble-t-il, de la transgression déontologique indéniable qu'ils accomplissent, le patient fût-il consentant? (On ne peut que s'interroger sur les effets d'un tel "consentement") Comme si 
l'interdiction de s'exposer personnellement, interdiction à laquelle, nous l'avons vu, certains contreviennent, se trouvait chez d'autres allégée par l'exposition de leurs patients à laquelle ils procèdent...

Voilà, on l'espère, qui fait aller de soi (justement) une question que l'on aurait pu poser d'emblée: de quel "soi "s'agit-il? Depuis l'invention de la psychanalyse par Sigmund Freud - mais il ne faut pas oublier qu'elle a eu lieu et temps à une époque où les limites du "soi " recevaient d'autres coups (voir par exemple à ce sujet le livre de Jacques Le Rider, Modemité viennoise et crises de l'identité-9) - le "soi" n'est plus précisément défini: les incertitudes de sa nomination augmentées par les difficultés de traduction (qu'est-ce que le "soi" dans l'écriture allemande donc de Freud? Qu'est-ce que le "self " par rapport au soi par exemple?) l'ont fait vaciller; puis les variantes de la topique freudienne: qu'est donc le soi si l'appareil psychique est constitué d'un "moiflanqué-d'un-surmoi-sous-tendu-par-un-ça"? Puis, si l'on veut bien qu'il subsiste, les jeux d'influences dont il est le thêâtre, les "identifications" qui le travaillent, les processus inconscients dont il est le siège sans en être le maître, mêlent à son aspect "actif" une allure passive qui l'altère, au sens fort du terme: c'est-à-dire que son identité est entamée par les marques de l'autre qu'il porte souvent à son insu, et supporte parfois bien malgré lui lorsqu'il les a repérées, rejetant avec obstination certaines ressemblances qu'on lui assigne, défendant comme il le peut son narcissisme en élisant parmi ceux qu'on a pu appeler "Les visiteurs du Moi " ${ }^{10}$ les images, les représentations, les personnages qui lui conviennent et qu'il intègre parmi les composantes de sa "personnalité ".

Bref, si l'on admet ce pluriel intérieur, n'est-on pas tenté de glisser, sans affirmation d'une différence radicale, du récit de soi au récit de l'autre, d'adopter des places d'écriture différentes pour parler de soi mais aussi d'"autour de soi", en somme, de passer de l'auto-biographie au roman sans modification profonde de posture? On pourrait reconsidérer de ce point de vue et pour ce qui concerne les textes freudiens des ouvrages comme L'interprétation des rêves, les "cinq psychanalyses" (qui d'ailleurs ne 
74

forment qu'un tout artificiel) et L'bomme Moise et la religion monotbéiste en se rappelant, si l'on admet le caractère autoanalytique du premier ouvrage reconnu par son auteur, les qualificatifs que Freud attribuait aux autres: "Krankengeschichte" "histoires de malades" et "roman historique". La fiction est bien proche du vrai, de la réalité. Et si le mot "roman" est dans le dernier cas une mesure de prudence, il est peut-être aussi le témoin d'une nostalgie. Il fallait sans doute à Freud tout son attachement à la science pour ne pas basculer du côté où il avait aussi rêvé d'être: celui de l'écrivain. Renoncement que vint d'ailleurs malgré tout (malgré son choix scientifique) adoucir le prix Goethe qui fut attribué à l'auteur.

Si bien qu'on pourrait proférer après tout, d'un point de vue littéraire: "Mö̈se, c'est Freud" comme Gustave Flaubert lançait: "Madame Bovary, c'est moi." N'est-ce pas là alors une piste qui s'ouvre à l'écriture psychanalytique? Un moyen de lui faire prendre conscience que, derrière l'observation d'un "cas", et outre le désir de parler d'un soi masqué qu'on a signalé plus haut, c'est une activité fictionnelle qui s'exerce à bas bruit, dûe au caractère individuel de l'interprétation chez l'auditeur-narrateurpsychanalyste, à la part de construction qu'il ajoute toujours à ce qui se voudrait une description? Il se pourrait que l'on doive être à la fois plus rigoureux et plus libre. L'on aurait à accepter que l'écriture d'héritage médical n'ait qu'une apparence scientifique pour camoufler ce qui recouvre une histoire doublement personnelle quant au conteur et quant au conté. Il est douteux d'ailleurs, plus largement, que les catégories médicales puissent s'appliquer à l'exercice psychanalytique, qu'elles lui soient pertinentes ou profitables en tout cas; probable au contraire que l'objectivité qui veut avoir présidé au choix du matériel ne soit qu'illusoire, dissimulant à son insu l'inéluctable sélection que tout lecteur, tout auditeur effectue. Le sort des cinq grandes observations de Freud est de ce point de vue assez remarquable: illustrant des entités cliniques comme le manifestent leurs titres respectifs, elles en sont venues, avec le temps, le recul historique, le poids de la littérature spécialisée qui les a étudiées, à n'être plus évoquées pratiquement que par le nom - ou le surnom de leur "héros": Dora, l'Homme aux rats, l'Homme aux loups, le Président Schreber, le petit Hans. On serait chez Jack London... ou Balzac et, à travers tel ou tel auteur, on suivrait les aventures de l'inventeur de la psychanalyse. 
On voudrait alors, puisque le récit de soi est inévacuable, que le "pluriel intérieur" imposé par la découverte freudienne trouve intérêt à se refléter dans des écritures multiples, adoptant des places multiples ${ }^{11}$. Une chose est certaine en tout cas: la séance, le régime, l'allure de la séance, ne sauraient se traduire dans la forme classique, narration d'une séquence de la cure, "vignette clinique" puis développement théorique la concernant. Cette figure de style ne rend pas compte de la position respective des protagonistes, beaucoup plus intriquée, sujette à des permutations imaginaires nécessaires, que l'écrit traditionnel ne veut bien le dire. C'est le passage par la psychanalyse personnelle qui impose ces détours par l'autre, même si son récit de vie est différent, même si ses formations névrotiques n'adoptent pas les mêmes contours que celles de l'analyste: les thèmes fantasmatiques se retrouvent dans leurs grandes lignes mais, alors que les détails individuels sont toujours différents (heureusement pour le praticien!), leur travail dans l'inconscient, leurs tracés dans l'appareil psychique sont comparables de l'un à l'autre. Ce qui revient à accepter, dès que l'on veut écrire de ces processus, un minimum de récit de soi. Étant entendu que le récit de soi de l'analyste devrait se tenir au minimum...

On aurait aussi à examiner comment l'écriture psychanalytique reflète l'organisation socioprofessionnelle dans laquelle elle s'insère: écriture médico-psychologique, donc, traduisant l'origine majoritaire des praticiens de l'analyse, et plus ou moins influencée par des displines au discours plus serré comme la philosophie qui, elle aussi, fournit un contingent, plus restreint, d'analystes. Poids de l'institution universitaire, s'accroissant, en tout cas en France ces dernières années, au fur et à mesure que l'enseignement de la psychanalyse se développe dans les facultés. Si bien qu'à la forme "mémoire d'adhésion" que les groupes psychanalytiques autonomes demandaient déjà s'ajoute celle des co'sies d'examen qui scandent le processus d'obtention des diplômes. Autant de "peaux" (de parchemins ou de peaux d'ânes) mal adaptées au "compte rendu" de ce qu'on entend dans la séance, alourdissant le moule de rationalité que la scolarité, largement répandue dans les pays où se pratique la psychanalyse,

11 Je me permets à ce propos de renvoyer à mon livre, "Tu as changé...", Paris, Aubier, 1987, dans lequel j'ai tenté une exploration des places différentes où écrire du processus psychanalytique en tant qu'acteur. 
impose aux enfants. Nécessaire certes, à leur développement mental et à leur future insertion dans la société, mais inhibant aussi dans certains cas leurs capacités d'invention, comme on le voit si bien à travers les productions de la période de latence. La puberté est loin de réussir toujours à lever le couvercle posé sur la créativité et, pour ceux qui arrivent sur le divan, c'est une découverte d'accession difficile que l'"association libre".

Bien sûr, on en connait les limites, on sait l'ambiguïté de cette liberté-là, comme de toute liberté du reste. Mais elle est quand même à mon sens un des apports essentiels de la cure, susceptible de lever les inhibitions douloureuses alimentées par des culpabilités fondamentales (toutes celles qui affectent la jouissance solitaire, les jouissances solitaires sur lesquelles plane l'ombre de la masturbation) interdisant tout "parler de soi " et prescrivant le secret sur des transgressions dramatisées aux yeux du sujet qui les percevra par la suite comme les plus banales du monde. Que le passage de ce qui a été soigneusement caché pendant des années à la possibilité du dire sans châtiment ait pu faire considérer la psychanalyse comme une technique de l'aveu, ainsi qu'on le lit dans Michel Foucault ${ }^{12}$, reflète peut-être une vue qui s'ancre elle-même dans des pratiques religieuses traditionnelles plutôt qu'elle ne les décèle à bon droit dans le parcours psychanalytique: il ne s'agit pas par la cure, me semble-t-il, de découvrir une vérité cachée du sujet, clé magique en quelque sorte de sa névrose (vue à laquelle il adhérerait volontiers, car elle est plus conforme à l'imaginaire infantile) mais d'instaurer un parcours de sens (au pluriel); tout au long de celui-ci, et si l'on voulait encore adopter un vocabulaire de l'enfermement, on procéderait à une "levée d'écrou", une "relaxe", par mise en évidence de l'inconsistance des motifs de la détention.

Ce qu'on pourrait donc souhaiter à l'écriture psychanalytique du récit de soi, pour peu qu'elle s'y aventure - on a bien vu que ce texte est plutôt un exposé de mes réticences, ou après tout "résistances" à m'y engager qu'un encouragement à l'entreprise c'est en tout cas de refléter cette conquête: de renoncer, en priorité à la confortable linéarité qui mène, le long d'une chronologie solidement tenue, d'un début, obligatoirement reconstitué à l'aide

12 Michel Foucault, Histoire de la sexualité. 1 La volonté de savoir, Paris, Gallimard, 1976. 
des souvenirs d'autrui, jusqu'à l'ultime moment, que ce soit celui du temps où s'écrit le dernier chapitre provisoire, ou bien celui que l'irruption de la mort viendra signifier. Elle devrait oser une réticulation plus en accrd avec les multiples temps qui jouent dans l'analyse, et même au risque de s'y perdre parfois. Adopter les changements de registre qui marquent, dans les bons cas, la circulation de l'analysant en ses discours à travers ses différents espaces psychiques: celui du rêve, du souvenir, de la chronique d'actualité. Comme en travaillant un dessin dans lequel les contours du modèle ne s'établissent qu'à l'aide d'une multiplicité de traits, de prises et de reprises, de saisies et de déssaisies (voyez Giacometti), on essaierait de parvenir à des décollements progressifs des variantes du roman familial à partir de son modèle initial, porteur de la décompensation névrotique qui a suscité la cure. Il faudrait aussi se ménager (mais n'est-ce pas déjà un terme trop volontariste?), se faire à des surprises, celles mêmes sans lesquelles il n'y a pas, à proprement parler, de psychanalyse, non seulement du côté du psychanalysant mais encore du psychanalyste, comme en témoigne si bien l'ouvrage de Theodor Reik intitulé Le psycbologue surpris ${ }^{13}$. L'inconscient est bon garçon, somme toute: il n'est pas avare de ces surprises-là, pour peu qu'on veuille bien les accueillir après qu'on ait fait leur connaissance en cours de cure et que, la porte du psychanalyste une fois refermée, on ne leur condamne pas la sienne propre. Ce sont elles qui conduisent aux mini-découvertes personnelles dont le plaisir nous est laissé, après le grand défrichement freudien initial, fixation d'un cadre minimum du savoir qui n'est certes pas intangible, mais qu'il faudrait s'occuper de raffiner plutôt que de complexifier à l'excès sans que la nécessité en ressortisse à l'écoute analytique... En ce sens, l'écoute à soi en tout cas est nécessaire, à condition qu'elle ne retombe pas, la brèche qu'a pratiquée l'analyse n'ayant que trop tendance à se colmater, dans la pratique introspective dont justement le propos de l'analyse est qu'elle soit abandonnée. Comment instaurer, maintenir la mise en déséquilibre d'un discours jusque là assuré de ses assises, c'est la question qui, schématiquement travaille l'écrit de l'analyste sur soi, pour peu qu'il veuille témoigner de son expérience spécifique. Du moins devrait-il en laisser passer le reflet.

13 Theodor Reik, Der überrascbte Psychologe, Leiden, 1935. Le psychologue surpris, Paris, Denoël, 1976 pour la trad. franç. 
Mais un autre aspect encore doit nous occuper avant de terminer: celui des rapports du récit de soi avec la mort. L'on sait, de façon générale, qu'ils sont étroits. D'une part, l'autobiographie est un moyen supplémentaire de garantir sa survie, de conférer à celle-ci une signature avec, au moins, les grandes lignes d'une identité, de camper une personne derrière une œuvre, de donner à savoir à qui l'on a affaire lorsqu'on la lit et, dans le cas particulier de l'analyste, de dévoiler aux ex-patients, au cas où ils s'y intéresseraient encore après-coup, de quoi satisfaire une curiosité restée inassouvie. Démarche incertaine quant à la justification de ses effets, me semble-t-il: l'"humanité " du psychanalyste derrière son patient, sa destitution nécessaire de la position de sujet supposé savoir, donc tout-puissant, qui lui était prêtée, passe par d'autres voies que les précisions autobiographiques des événements de sa vie, de ses goûts et intérets, voire de ses amours et désamours. Si on la tente, n'est-ce pas, en quelque sorte, pour contracter une assurance vie-posthume établissant le lien entre l'homme et l'œuvre lien dont on connaît pourtant le caractère discuté? Pour prolonger sa présence à partir de l'au-delà et "doubler" de son "soi " ce que l'on a créé? Étoffer d'avance - et quoi de plus compréhensible - l'inscription qui figurera sur la pierre tombale? Et, à l'autre bout du fil - la naissance —, aménager son propre commencement autant, aussi loin qu'il est possible de le faire?

Autre type de garantie: "méfiez-vous des contrefaçons"! Rappelons-nous la sévère mise en garde que Freud adressait à Arnold Zweig tout prêt à écrire la biographie du fondateur de la psychanalyse au moment du quatre-vingtième anniversaire de celui-ci: "Non, je vous aime trop pour vous permettre une chose pareille. Celui qui devient biographe s'oblige au mensonge, aux secrets, à l'hypocrisie, à l'idéalisation et même à la dissimulation de son incompréhension, car il est impossible d'avoir la vérité biographique, et même si on l'avait, elle ne serait pas utilisable." Soit. Nous y sommes à peu près préparés. Mais la suite est plus curieuse: "La vérité n'est pas praticable, les hommes ne la méritent pas, et d'ailleurs notre prince Hamlet n'a-t-il pas raison lorsqu'il demande si quelqu'un pourrait échapper au fouet s'il était traité selon ses mérites?" "14 Certes, il s'agit là d'une vue sans indulgence de la nature humaine. Mais, si l'on suit Freud, ne 
tenterait-on pas, en écrivant son autobiographie, et a fortiori lorsqu'on est psychanalyste, c'est-à-dire en partie conscient de ses abîmes psychiques, d'éviter le fouet que vous administreraient les autres, ou du moins d'en adoucir la correction? Le récit de soi constitue à cet égard un manteau protecteur efficace, du moins contre les premiers coups. Avec, si j'ose dire, la bénédiction de la déculpabilisation psychanalytique... 